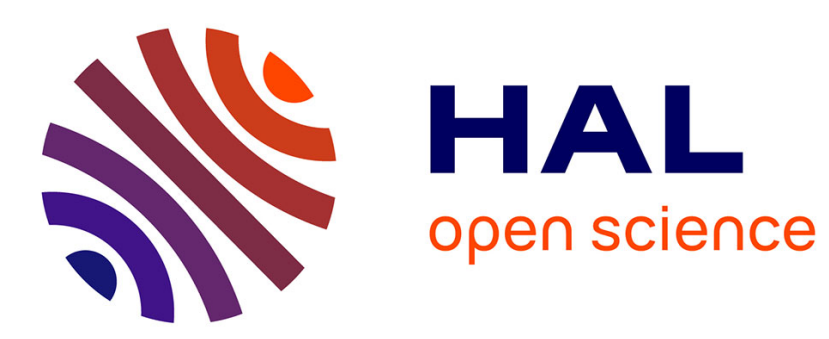

\title{
Viscosity and reptation time in polystyrene-benzene semidilute solutions
}

\author{
M. Adam, M. Delsanti
}

\section{To cite this version:}

M. Adam, M. Delsanti. Viscosity and reptation time in polystyrene-benzene semidilute solutions. Journal de Physique Lettres, 1979, 40 (19), pp.523-527. 10.1051/jphyslet:019790040019052300 jpa00231679

\section{HAL Id: jpa-00231679 https://hal.science/jpa-00231679}

Submitted on 1 Jan 1979

HAL is a multi-disciplinary open access archive for the deposit and dissemination of scientific research documents, whether they are published or not. The documents may come from teaching and research institutions in France or abroad, or from public or private research centers.
L'archive ouverte pluridisciplinaire HAL, est destinée au dépôt et à la diffusion de documents scientifiques de niveau recherche, publiés ou non, émanant des établissements d'enseignement et de recherche français ou étrangers, des laboratoires publics ou privés. 


\title{
Viscosity and reptation time in polystyrene-benzene semidilute solutions
}

\author{
M. Adam and M. Delsanti \\ Service de Physique du solide et de Résonance magnétique, DPh-G/PSRM, CEN, Saclay, B.P. nº 2, 91190 Gif sur Yvette, France \\ (Reçu le 27 juin 1979, accepté le 21 août 1979)
}

Résumé. - Nous donnons quelques résultats expérimentaux préliminaires concernant la viscosité et le temps de reptation de solutions semi-diluées (polystyrène benzène).

Nous avons obtenu les résultats suivants :

et

$$
\eta \sim M^{3 \mp 0,5} c^{4,5 \mp 0,1}
$$

$$
T_{\mathrm{R}} \sim c^{2,8 \mp 0,4} .
$$

Les valeurs des exposants de concentration ne sont pas en accord avec celles prévues par de Gennes.

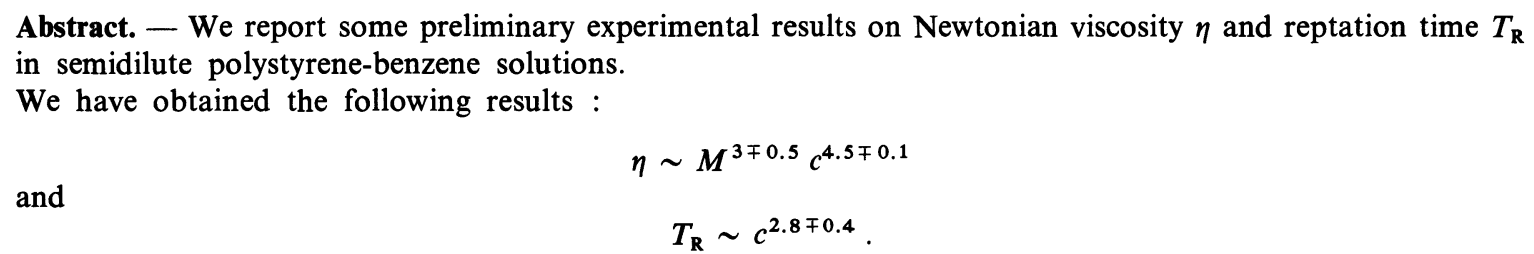

The concentration exponent values are not in agreement with de Gennes' theory.

It has been shown experimentally by many authors $[1,2,3]$, that the viscosity of a concentrated solution of polystyrene in various good solvents can be described as :

$$
\begin{array}{lll}
\eta \sim M & \text { if } & M<M_{\mathrm{c}} \\
\eta \sim M^{3.4} & \text { if } & M>M_{\mathrm{c}}
\end{array}
$$

$M$ is the molecular weight of the macromolecule and $M_{\mathrm{c}}$, the critical molecular weight for entanglements, is of the order of $2 \times 10^{4}$ daltons.

One of these authors has shown [2] that :

$$
\eta \sim(c[\eta])^{4.7}
$$

where $[\eta] \sim M^{0.72}$ is the intrinsic viscosity and $c$ the monomer concentration.

Recent experimental and theoretical work [4] has shown the existence of three concentration regimes in polymer solutions : dilute, semidilute and concentrated. Here, our aim is to study the viscoelastic properties of polystyrene-benzene systems in a well defined semidilute regime. We recall briefly the conformation of chains in semidilute solution [4] and theoretical predictions on their dynamical properties [5], and then, we give the description of the experiments and the experimental results obtained.

Let us consider a solution whose monomer concentration is higher than the overlap concentration, $c^{*}$, defined as :

$$
c^{*} \simeq M / A R^{3} \sim M^{-0.8}
$$

$A$ is the Avogadro number, $R$ is the radius of gyration of an isolated macromolecule $\left(R \sim M^{v}, v=0.6\right.$ in good solvent). The semidilute regime is meaningful if the monomer concentration, $c$, follows the condition :

$$
c^{*} \ll c \ll c_{\mathrm{s}}
$$

where $c_{\mathbf{s}}$ is the solvent concentration.

Then the macromolecules interpenetrate each other and have contact points; $\xi$, is the mean distance between these points and $T_{\mathrm{R}}$ their finite life time.

The length $\xi$, derived from an analogy with critical 
phenomena, is molecular weight independent and decreases with concentration as :

$$
\xi \sim c^{-0.75} .
$$

This law has been checked experimentally by elastic neutron scattering [4].

The life time $T_{\mathrm{R}}$ is the time required for one macromolecule to change its conformation amongst the others. $T_{\mathrm{R}}$ has been calculated assuming that the molecule reptates along a virtual tube $[5,6,7]$ of diameter $\xi$ and length $[M / M(\xi)] \xi$. $M(\xi)$ is the molecular weight of a part of chain between two contact points.

This leads to :

$$
T_{\mathrm{R}} \sim M^{3} c^{1.5}
$$

Now, let us consider the viscoelastic properties of such a system. At a time scale smaller than $T_{\mathrm{R}}$ but much bigger than $T_{\xi}$, it has a finite elasticity and thus behaves like a gel of mesh size $\xi-T_{\xi}$ is the time corresponding to the mesh first mode. The shear elastic modulus $G_{0}$ is proportional to the number of contact points per unit volume.

$$
G_{0} \simeq \frac{k T}{\xi^{3}} \sim c^{2.25}
$$

On a time scale larger than $T_{\mathrm{R}}$ the liquid is Newtonian with a viscosity $\eta$. From a dimensional argument [8] and for continuity between the two behaviours, we have :

$$
\eta \simeq G_{0} T_{\mathrm{R}}
$$

which becomes using expressions (3), (4) and (1) :

$$
\eta \sim M^{3} c^{3.75} \sim\left(\frac{c}{c^{*}}\right)^{3.75}
$$

In terms of phenomenological viscoelastic theory, this behaviour corresponds to a Maxwell model with a relaxation modulus [9] :

$$
G(t)=G_{0} \mathrm{e}^{-t / \widetilde{T}_{\mathrm{R}}} .
$$

$\tilde{T}_{\mathrm{R}}$ is the relaxation time of a stress when a sudden strain is imposed. In our case, we assume that $\tilde{T}_{\mathrm{R}}$ is proportional to the life time $T_{\mathrm{R}}$, which is the longest time in the semidilute solution.

Since, for small strain and simple shear, the effects of mechanical history are linearly additive, the stress is given by :

$$
\sigma(t)=G_{0} \int_{-\infty}^{t} \exp \left\{-\frac{t-t^{\prime}}{\tilde{T}_{\mathrm{R}}}\right\} \dot{\gamma}\left(t^{\prime}\right) \mathrm{d} t^{\prime}
$$

where $\dot{\gamma}$ is the shear rate.

Measurements of stresses and strains allow the viscosity and the relaxation time, $T_{\mathrm{R}}$, of the semidilute solution to be determined.
In our experiments we measure the shear stress with the help of a magnetic sphere immersed in the solution. The principle of the apparatus [10] is schematically represented in figure 1 . The sphere is located in a region where the magnetic field created by a coil is not uniform and thus it experiences a magnetic force. The current intensity, $I$, in the coil is adjusted in such a way that the sphere holds at a fixed position when the solution is displaced by $\delta$.

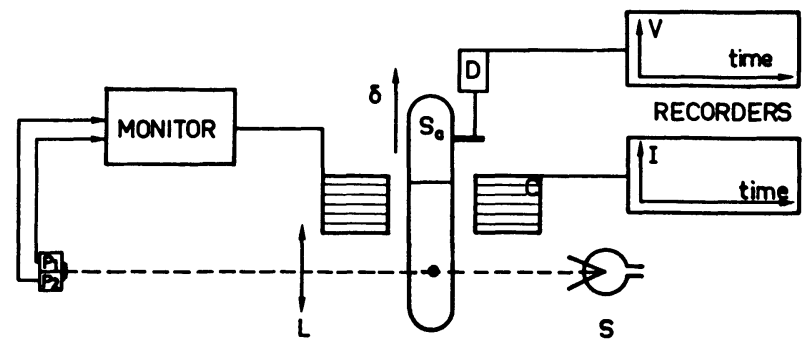

Fig. 1. - By means of light source, $S$, and a lens, $L$, the image of the sphere projected to the surface of the photodiodes $P_{1} P_{2}$. The differential current of photodiodes $P_{1} P_{2}$, is fed to the monitor which adjusts the intensity current, $I$, of the coil, $\mathrm{C}$, in such a way that the sphere remains at the same place in the cell $(\mathrm{Sa})$ which contains the polymer solution studied. The displacement, $\delta$, is measured by recording the output voltage of a displacement detector, $\mathrm{D}$.

We measure the intensity $I$ and the displacement $\delta$ which are proportional to $\sigma$ and $\gamma$ respectively.

The viscosity of the solution is obtained if a constant shear rate $\dot{\gamma}$ is applied at time 0 . From the expressions (8) and (5) we have :

$$
\sigma=G_{0} \tilde{T}_{\mathbf{R}} \dot{\gamma}=\eta \dot{\gamma} \quad \text { if } \quad t \gg \tilde{T}_{\mathbf{R}}
$$

which corresponds in our experiments to :

$$
\eta=\alpha(I / \dot{\delta})
$$

where $I$ is the stationary magnetic coil intensity measured at a time much longer than $T_{\mathrm{R}}\left(t>5 \widetilde{T}_{\mathrm{R}}\right)$, and $\alpha$ is a factor related to the magnetic moment of the sphere and to the geometry of the experiment. In order to determine the factor $\alpha$, we also measure $I / \dot{\delta}$ for a reference solution of viscosity $\eta_{\mathrm{r}}$ in the same experimental conditions. This allows us to obtain the relative viscosity $\eta / \eta_{\mathrm{r}}$.

As shown in figure 2, for each solution, we verify that the viscosity is Newtonian.

This apparatus allows one to measure viscosities ranging from 0.3 poises to $10^{4}$ poises, reproductible to about $8 \%$.

The viscosity of polystyrene-benzene solutions is studied as a function of the concentration for different molecular weights ranging from $4 \times 10^{6}$ to $24 \times 10^{6}$ daltons, at $32^{\circ} \mathrm{C}$. The concentration range investigated corresponds to $c / c^{*}>4$ and $c$ smaller than $10^{-1}(\mathrm{~g} / \mathrm{g})$ in order to satisfy the inequality 


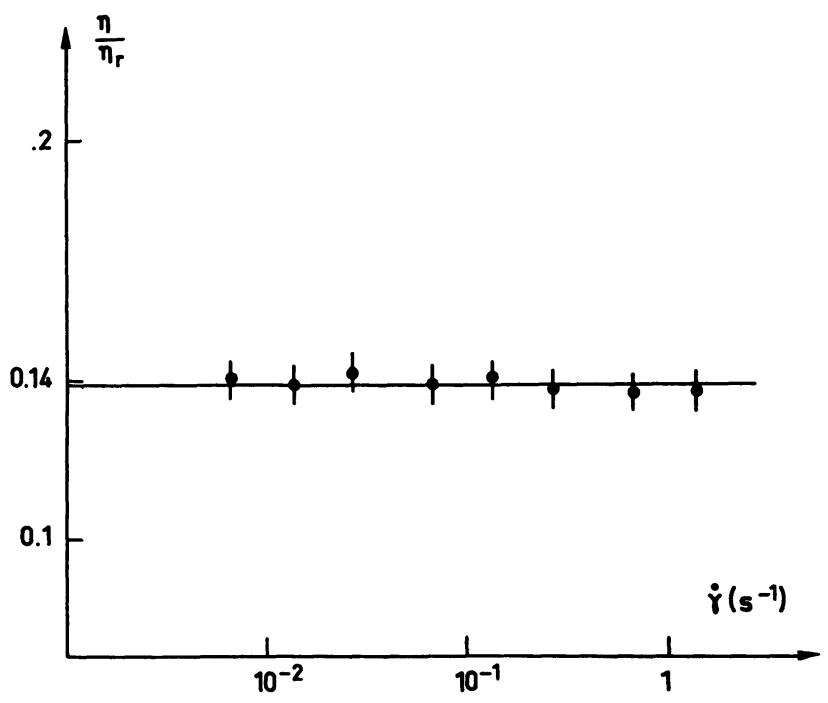

Fig. 2. - Relative viscosity versus shear rate $\dot{\gamma}$ defined as : $\dot{\gamma}=\dot{\delta} / a$, where $a(\approx 0.5 \mathrm{~mm})$ is the radius of the sphere.

$c^{*} \ll c \ll c_{\text {s. }}$. The $c^{*}$ values for polystyrene-benzene systems are determined using the expression given in reference [11]. The characteristics of the samples and the viscosity measurements are listed in table I and reported on a log-log scale in figure 3 .

For $c / c^{*}>7$, it is found that the viscosity behaviour can be described by a power law :

$$
\frac{\eta}{\eta_{\mathrm{r}}}=F(M) c^{x_{\eta}}
$$

where the prefactor $F(M)$ depends only on the molecular weight.

As shown in table II, a least square fit gives

$$
x_{\eta}=4.5 \mp 0.1
$$

which is larger than the exponent obtained from the reptation model (eq. (6)) but compatible with exponent

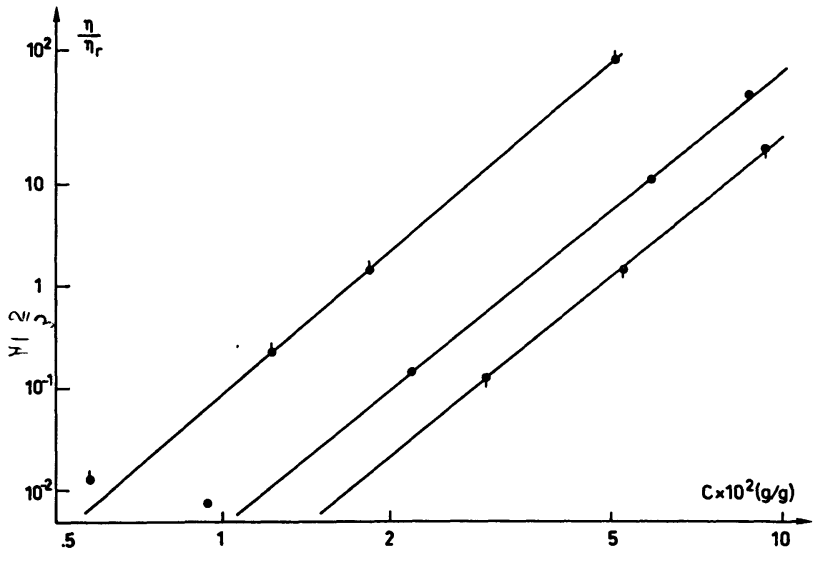

Fig. 3. - Variation of the relative viscosity versus concentration for the 3 molecular weights used :

- $M_{\mathrm{w}}=24 \times 10^{6}, \bullet M_{\mathrm{w}}=7.1 \times 10^{6}, \bullet M_{\mathrm{w}}=4.1 \times 10^{6}$.

Table II. - Calculated values of $F(M)$ and the concentration exponent $x_{\eta}$ defined in the text.

\begin{tabular}{llc}
\multicolumn{1}{c}{ Sample } & \multicolumn{1}{c}{$F(M)$} & $x_{\eta}$ \\
$1,2,3$ & $(1.44 \pm 0.02) 10^{8}$ & $4.61 \pm 0.01$ \\
$5,6,7$ & $(3.27 \pm 0.6) 10^{6}$ & $4.44 \pm 0.06$ \\
$9,10,11$ & $(7.34 \pm 1) 10^{5}$ & $4.43 \pm 0.07$
\end{tabular}

values previously found from experiments in concentrated regime [2].

For $c / c^{*}<7$ the experimental points deviate systematically from this law.

If we assume that the prefactor $F(M)$ varies with the molecular weight (Fig. 4) as a power law : $F(M) \sim M^{x}$, we obtain :

$$
x=3 \pm 0.5
$$

the poor accuracy of this exponent is due to the high polydispersity of the samples.

Table I. - Characteristics of the samples used and experimental results on viscosity and relaxation time. The symbols used are defined in the text, except $M_{\mathrm{w}} / M_{\mathrm{n}}$ which is the polydispersity index as given by the supplier and $\eta_{\mathrm{r}}$ which is the viscosity of the reference solution (silicon oil, $\eta_{\mathrm{r}} \approx 50$ poise).

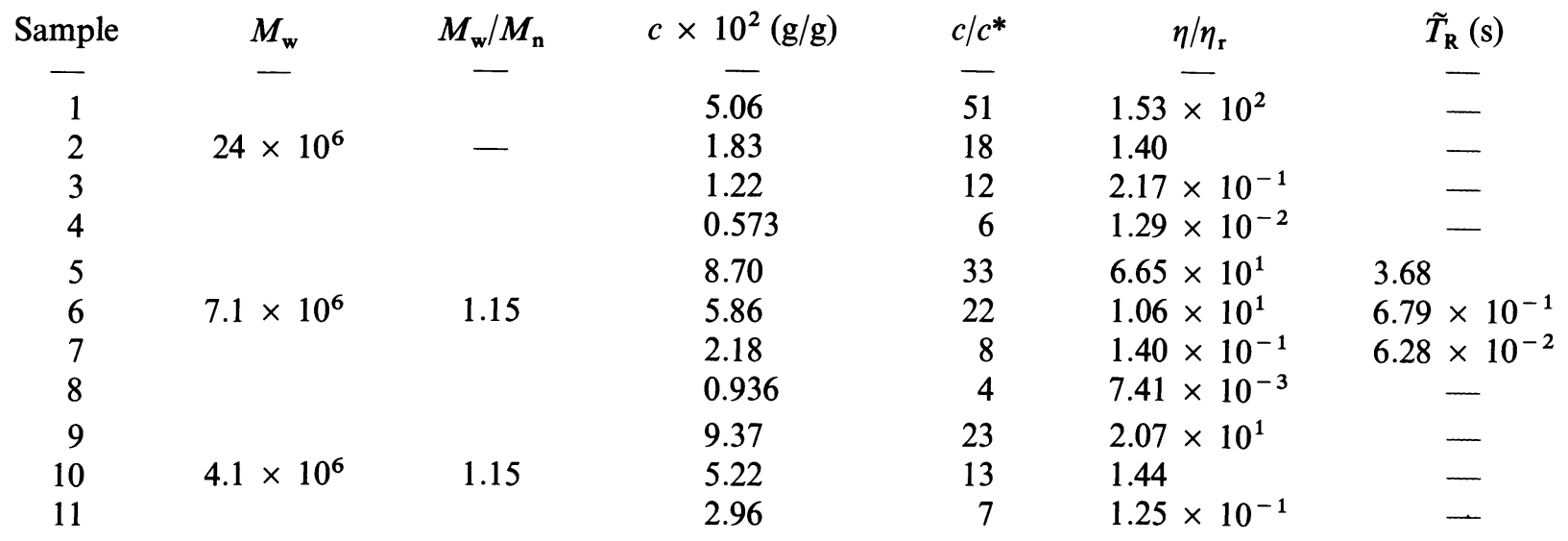




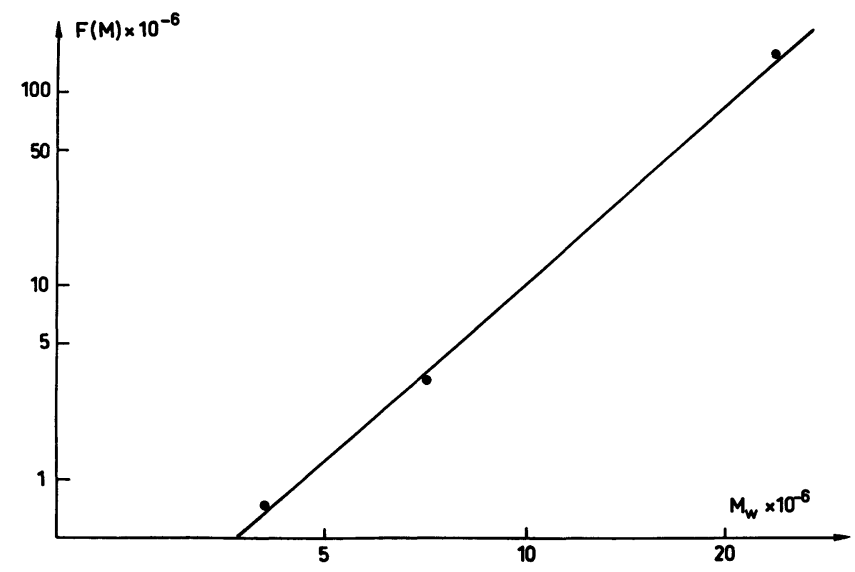

Fig. 4. - Variation of the prefactor $F(M)$ as a function of the molecular weight.

The exponent found lies between the value given by the theory [5] (eq. (6)) and that determined experimentally [1] $(x=3.4)$.

The relaxation time $\tilde{T}_{\mathrm{R}}$ is obtained if a constant shear strain $\gamma$, is imposed at a time 0 , during a period $\varepsilon(\approx 50 \mathrm{~ms})$ much shorter than $\tilde{T}_{\mathrm{R}}$, for which equation (8) becomes

$$
\sigma(t)=G_{0} \cdot \gamma \cdot \mathrm{e}^{-t / \widetilde{T}_{\mathbf{R}}} .
$$

which corresponds in our experiment to :

$$
I(t)=I_{0} \mathrm{e}^{-t / \widetilde{T}_{\mathbf{R}}} \quad \text { if } t \gg \varepsilon
$$

where $I_{0}$ is proportional to the shear modulus multiplied by the displacement.

Fitting the time dependence of the coil intensity $I(t)$ to an exponential function of time we determined $\tilde{T}_{\mathrm{R}}[12]$. $\tilde{T}_{\mathrm{R}}$ has been studied (table I) for a given molecular weight $-7.1 \times 10^{6}$ daltons - as a function of the concentration $\left(c / c^{*}>8, c<10^{-1} \mathrm{~g} / \mathrm{g}\right)$

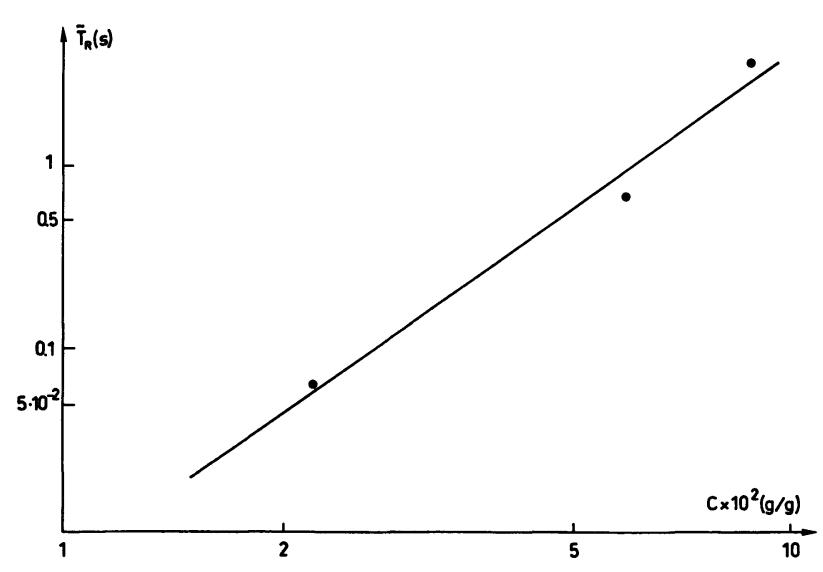

Fig. 5. - Variation of the relaxation time as a function of concentration. at $32^{\circ} \mathrm{C}$. The experimental points $\tilde{T}_{\mathrm{R}}$, plotted on a $\log -\log$ scale (Fig. 5) can be approximated to the power law :

$$
\tilde{T}_{\mathrm{R}} \sim c^{x_{\mathrm{T}}} \quad \text { with } \quad x_{\mathrm{T}}=2.8 \mp 0.4
$$

which is larger than the theoretical value 1.5 [5]. One must compare our exponent value $x_{\mathrm{T}}$, to that determined by using a forced Rayleigh scattering technique [13], which, via concentration dependence of the self diffusion coefficient $\left(D_{\mathrm{S}} \sim c^{1.7 \mp 0.1}\right)$ yields $T_{\mathrm{R}} \sim c^{1.5}$, in agreement with theoretical prediction. We have no satisfactory explanation concerning the discrepancy between the results given by these two methods.

From the general relation $\eta=G_{0} \tilde{T}_{\mathrm{R}}$ and the concentration exponents $x_{\eta}, x_{\mathrm{T}}$ we can deduce the exponent of the shear modulus :

$$
G_{0} \sim c^{x_{\mathrm{G}}} \text { with } x_{\mathrm{G}}=1.7 \mp 0.5 .
$$

This exponent is compatible with theory and mechanical measurements on polystyrene gel swollen by benzene [14].

These experiments, performed on systems which corresponds to the hypothesis of de Gennes' theory, show clearly that the viscosity of a semidilute polystyrene-benzene solution does not obey to the scaling law $: \eta \sim\left(c / c^{*}\right)^{3.75}$. However the relation $\eta \simeq G_{0} T_{\mathrm{R}}$ seems to be valid.

It is useful to note here, that, one of the experiments [15] done on semi-dilute polyisobutylene (PIB) toluene solutions $(c<10 \%)$ yields :

$$
\eta \sim M^{2.9 \pm 0.4} c^{3.8 \mp 0.2}
$$

in agreement with expression (6).

However, the two systems (PIB toluene-PS benzene) present a number of mismatching features (such as : quality of the solvent, flexibility of the macromolecule, glass temperature transition of the melt, value of $M_{\mathrm{c}}$ ) which may result in somewhat different respective values of the exponents $x_{\eta}$ and $x_{\mathrm{T}}$.

On the other hand, by some improvements of the apparatus it should be possible to measure the shear modulus and to determine its variation as a function of both concentration and molecular weight. By increasing the $\tilde{T}_{\mathrm{R}}$ precision we hope to be able to determine the origin of the discrepancy between experiments and theory for the viscosity of polystyrene-benzene semidilute solutions.

Acknowledgments. - We are indebted to P. Pieranski for his helpful assistance and for making available the magnetic rheometer apparatus. We wish to thank C. Williams, G. Jannink and P. G. de Gennes for stimulating discussions. 


\section{References and notes}

[1] Berry, G. C., Fox, T. G., Adv. Polym. Sci. 5 (1968) 261.

[2] Onogi, S., Kimura, S., Kato, T., Masuda, T., Miyanaga, N. J. Polym. Sci. Pt C 15 (1966) 381.

[3] Dreval, K. E., Botvinnik, G. O., J. Polym. Sci. (Polymer Physics ed.) 11 (1973) 1055.

[4] Daoud, M., Cotton, J. P., Farnoux, B., Jannink, G., Sarma, G., Benoit, H., Duplessix, R., Picot, C. and DE Gennes, P. G., Macromolecules 8 (1975) 804.

[5] DE GenNes, P. G., Macromolecules 9 (1976) 587.

[6] KLein, J., Nature 271 (1978) 143.

[7] De Gennes, P. G., Daoud, M., to be published in J. Polym. Sci.

[8] See for instance LANDAU and LifCHITZ. Elasticity (Pergamon Press, London) 1959.

[9] See for instance, FerRY, J. D., Viscoelastic properties of polymer (J. Wiley, New York) 1970, Chap. I.
[10] Meyer, R. and Pieranski, P., Preprint (P. Pieranski, Bât. 510, Univ. Paris-Sud F 91405 Orsay, Cedex).

[11] Adam, M. and Delsanti, M., Macromolecules 10 (1977) 1229. [12] In fact, we have $I(t)=I_{0} \mathrm{e}^{\left(-t / \widetilde{T}_{\mathrm{R}}+T_{\mathrm{a}}\right)}$ where $T_{\mathrm{a}}$ is the response time of the apparatus. We can deduce $T_{\mathrm{a}}$ from experiments on the reference solution which has no viscoelastic behaviour at the time scale considered. This experimental procedure will be given in details in a future paper.

[13] Hervet, H., Leger, L., Rondelez, F., Phys. Rev. Lett. 42 (1979) 1681

[14] BelKebir-Mrani, A., Thesis Strasbourg (1976).

Munch, J. P., CANDau, S., Herz, J., Hild, G., J. Physique 38 (1977) 97.

[15] Von Schurz, J. and Hochberger, H., Makromol. Chem. 96 (1966) 141. 